\title{
The maturing of therapy*
}

\section{Some brief psychotherapies help anxiety/depressive disorders but mechanisms of action are unclear}

ISAAC M. MARKS

\author{
Background Psychiatric therapy \\ needs assessment regarding its maturation \\ as a therapeutic science.
}

\begin{abstract}
Aims Judgement of whether such a science is emerging.

Method Four criteria are used: efficacy; identification of responsible treatment components; knowledge of their mechanisms of action; and elucidation of why they act only in some sufferers.
\end{abstract}

\section{Results Brief behavioural,} interpersonal, cognitive, problem-solving and other psychotherapies have a mature ability to improve anxiety and depressive disorders reliably and enduringly, often only with instruction from a manual or a computer. Therapy's cost-effectiveness and acceptability deserve more attention. We know little about which treatment components produce improvement, how they do so and why they do not help all sufferers.

\section{Conclusions Therapy is coming of age regarding efficacy for anxiety and depression, but is only a toddler regarding the scientific principles to explain its effects.}

\section{Declaration of interest I.M.M.} shares intellectual property rights in Fear Fighter, BTSteps and Cope.

\footnotetext{
*Based on the 16th Aubrey Lewis Lecture given at the Institute of Psychiatry, London, 14 December 2000.
}

The mental health field has a rich legacy from Sir Aubrey Lewis's vision of psychiatry as a behavioural science using 'empirical clinical methods strengthened by the results of research, which then enable theory to be formulated and eventually applied to practice' (cited by Shepherd, 1991). That vision of psychiatry as an empirical clinical science is becoming a reality, with collaborative research teams using standards of evidence that transcend any single country or school. Careful studies have yielded such a wealth of scientific findings and randomised controlled trials (RCTs) of treatments that Cochrane Collaboration groups have a hard task summarising their results. We can now improve anxiety, depressive and eating disorders, chronic fatigue and aspects of psychosis and unhealthy lifestyles far more than was possible in the 1960s. Can we therefore say that therapy has come of age, that there is a mature science of therapeutics?

\section{FOUR CRITERIA FOR MATURITY OF A THER APEUTIC SCIENCE}

Four criteria can be set to decide whether therapy has come of age:

Criterion 1: a given treatment must improve a particular problem (a) reliably, (b) enduringly and (c) cost-effectively and (d) must be acceptable to patients.

Criterion 2: only specified components of the treatment should produce the improvement.

Criterion 3: the mechanisms by which therapy components yield improvement can be delineated.

Criterion 4: why therapy components help only some sufferers and not others with the same problem can be pinpointed.

Few treatments in any branch of health care can survive all four tough tests. Do any in mental health care? For brevity this review will consider these criteria only for the treatment of anxiety and depressive disorders, and will dwell especially on Criterion 1 for whose four sub-criteria the evidence is richer than for the remaining three criteria.

\section{Criterion I, sub-criteria (a) and (b): the treatment must improve a particular problem reliably and enduringly}

\section{Depressive disorders}

There is little doubt that some brief psychotherapies and medications help depression sufferers reliably in the short term. Many RCTs found short-term relief from interpersonal psychotherapy, problem-solving, cognitive-behavioural therapy (CBT) and antidepressant medications. Short-term improvement of depression also was noted in the single RCTs that have tested other brief psychotherapies - the behavioural activation component of CBT without cognitive restructuring (Jacobson et al, 1996; Gortner et al, 1998), couple therapy for people with depression who live with a critical partner (Leff et al, 2000), aerobic exercise (Babyak et al, 2000) and mindfulness meditation for stress in volunteer students (Astin, 1997).

More problematic is the frequent relapse of depression after the end of treatment by medication or brief psychotherapy or both combined. Relapse may lessen by using CBT or behavioural activation alone (Fava et al, 1998; Gortner et al, 1998) or mindfulness meditation (Teasdale et al, 2000) or monthly maintenance interpersonal psychotherapy (Frank \& Spanier, 1995) or continuing medication. Improvement also was stable in the year after 15 hours of couple therapy ended in people with chronic depression living with a critical partner (Leff et al, 2000).

\section{Anxiety disorders}

In many RCTs of adults most anxiety disorders improved after brief psychotherapy in the form of exposure (Marks, 1987). In children, anxiety disorders were reduced and their onset was prevented by a mixed CBT and family-based group intervention (Barrett et al, 1996; Dadds et al, 1997). Moreover, improvement in chronic phobic and obsessive-compulsive disorders (OCDs) usually continued over 3- to 8-year follow-ups after the end of exposure therapy in England, Scotland, The Netherlands and 
Greece (O’Sullivan \& Marks, 1990). In Italy, enduring improvement of agoraphobia/ panic and of social phobia persisted over a mean of 7 years' follow-up after a therapist had spent 5-6 hours guiding self-exposure treatment (Fava et al, 2001a,b). Gains were especially stable if at pre-treatment patients had had no comorbid depression or personality disorder, and if by the end of treatment they had no residual avoidance and had given up benzodiazepines (Fava et al, 2001a,b). Relapsed patients usually improved again after brief booster selfexposure. About $30 \%$ of the Italian group with social phobia failed to complete treatment or benefited little from it.

\section{Do particular services yield reliable improvement year after year?}

Do services continue to help successive cohorts of patients reliably when they give treatment as a routine rather than in an RCT. The answer is yes for my residential Behavioural Psychotherapy Unit at the Bethlem Hospital, whose measures, types of patient (mainly with OCD) and kinds of treatment (usually self-exposure) remained comparable over the 11 years from 1987 to 1997. In four cohorts from that period (details available from the author upon request) roughly similar benefit was seen on ratings of the main problem and of work/social adjustment. Mean per-patient improvement hovered around $25-50 \%$ at discharge and at 3-6 months' follow-up. Few of the residential patients were cured completely and many did not improve. Those who failed with exposure also failed when cognitive therapy and/or medication were added. Most had very chronic and severe $O C D$, often with comorbid depression and personality disorders, all of which impair outcome. They had more severe illness and were more disabled and needed almost twice as much clinician time as my unit's out-patients with OCD did.

\section{Criterion I, sub-criteria (c) and (d): the treatment must improve a particular problem cost-effectively and must be acceptable to patients}

The fact that anxiety disorders usually remain improved for many years after the end of exposure therapy is vital, because most sufferers have had their problem for many years before entering RCTs. A treatment's value drops if improvement from it does not last, especially with chronic problems or if it must continue for years to avert relapse, unless the treatment is very cheap, easy to adhere to and without sideeffects.

Take the approximate costs in the USA of clomipramine or selective serotonin reuptake inhibitors (SSRIs) for OCD, including the cost of seeing a clinician to prescribe and monitor its effects, and of exposure therapy in my out-patients. We will ignore evidence that the drugs are slightly less effective than exposure at the end of treatment. A year after treatment began the cumulative costs of medication and of exposure therapies are comparable. However, the medication's cumulative cost rises over successive years as it continues under medical supervision to avert relapse. In contrast, the cumulative cost of one-off exposure rises little after the first year because most patients remain improved without needing boosters. Cost-effectiveness alters as treatment costs change. Clomipramine is cheaper in the UK than in the USA, and exposure costs less when guided by computer (see below).

The same considerations apply to other anxiety disorders, which usually have been very chronic in patients entering RCTs, and to sufferers of chronic depression. In the Leff et al (2000) study, the cost-effectiveness of desipramine and couple therapy were similar over 2 years. In an RCT for anxiety disorders in primary care, exposure therapy was cost-effective from the third year of follow-up onwards (Ginsberg et al, 1984). Regulatory bodies should demand evidence of cost-efficacy over years before approving a chronic treatment for a chronic disorder. The common assumption that pharmacotherapy costs less than psychotherapy in the long term is wrong where the psychotherapy is fairly brief and enduringly effective.

How acceptable sufferers find therapy deserves more attention by measuring their willingness to start, cooperate with and complete treatment, and preference for it when given an informed choice. Refusal and drop-out rates for psychotherapy and for medication often reach $50 \%$ but are usually lower in RCTs. In my out-patient behavioural unit for anxiety disorders, about $30 \%$ of patients refused to start or did not complete treatment over 28 years (1972-2000). Patients with anxiety disorder refuse to enter many RCTs because they object to medication more than they do to psychotherapy. In the Leff et al (2000) depression study, however, the drop-out rate was highest with cognitive therapy $(73 \%)$, then with desipramine $(57 \%)$ and lowest with couple therapy (15\%).

A treatment's acceptability is enhanced by educating patients about it, making it easier to do, reducing its side-effects and enabling sufferers to treat themselves.

\section{Reliable improvement with self-treatment}

There is mounting evidence from RCTs that sufferers' gains with CBT depend largely on their self-treatment done away from the therapist's presence. Emphasis in anxiety disorders has shifted from therapistaccompanied exposure towards selfexposure. Self-exposure is effective, for example, when guided by a live therapist face-to-face (Alkubaisy et al, 1992) or by telephone, and when it is guided by a manual sent by post (Burgess et al, 1998) or given by hand (Ghosh et al, 1988). Selftreatment guided by a manual also has helped bulimia and chronic fatigue.

Computer-guided self-help. Compared with self-treatment guided by post or a book, guidance by computer offers more interaction and individual tailoring and tracking of progress. 'Computer-guided therapy' here does not mean e-mail therapy, using the computer as a voice-mail system with a live human still giving the advice, but rather self-help by interaction with a computer system that itself asks the questions and uses patients' answers to direct the interview and give individually tailored advice. Research into computer-guided self-help is burgeoning and has promising outcomes.

Desk-top computer systems. Even with ancient computers from 1980, in RCTs sufferers of phobia and depression improved as much with computer-guided exposure or CBT as they did with face-to-face guidance (Ghosh et al, 1988; Selmi et al, 1990). Recently, in both a meta-analytical study and an RCT, phobia/panic sufferers improved as much after guidance mainly by a computer self-help system called FearFighter as after guidance entirely by a clinician (details available from the author upon request). Computer-guided users needed only about one-third of the clinician time required by patients who had no access to the system. A clinician raises productivity by delegating routine aspects of care to computer guidance and using his/her time to screen patients at the start 
and give brief back-up advice if required. A therapist thus can help several patients concurrently who use different computers simultaneously.

Many users value the confidentiality of computer-guided systems and confide sensitive information to a system that they would not confide directly to a human. They also prefer self-referral for computer guidance to avoid potential stigma from a professional referring them for a mental health problem.

Phone-accessed computer systems. Patients now access some computer guidance systems by phoning these from home. They drive the interview by pressing keys on their telephone keypad to activate an interactive voice response (IVR) system. Two such IVR self-help systems were developed by Dr John Greist in Madison, Wisconsin, Dr Lee Baer at Harvard University and myself in London.

Our first IVR self-help system was BTSteps $(\mathrm{BT}=$ behaviour therapy, not a phone company) for OCD. Patients are screened by a clinician on the phone or face-to-face and, if suitable (most are), they are given an identification number and a manual. They read a given step in the manual and then phone the computer from home to work out how to complete the selftreatment step. They do relevant homework assignments, read the next part of the manual and phone the computer again when ready for the next step or in need of further advice about the current step. The clinician is required only to screen patients and give occasional brief back-up advice by phone, and so can treat six times more patients with OCD per hour than without the system. A BTSteps Clinic at the Maudsley Hospital enrolled 120 patients with OCD from all over the UK for selftreatment by phoning from home.

BTSteps had promising results in a multi-site RCT in North America (Greist et al, 2002): 200 patients with OCD improved almost as much with computerguided exposure as with clinician-guided exposure, and both exposure groups improved more than with relaxation. In a meta-analysis, BTSteps completers improved even more than did treatment completers in the RCTs of SSRIs that persuaded the Food and Drug Administration to licence those drugs. In addition, in a small RCT at the Maudsley Hospital, patients with OCD showed bilateral caudate nucleus blood flow activation on a magnetic resonance imaging scan after improving by using BTSteps; this blood flow pattern had not been present before treatment began (details available from the author upon request).

The second IVR system, Cope, is for mild to moderate depression. Patients are provided with written workbooks to read about CBT before phoning the computer from home. In a meta-analysis, 41 people with depression in a pilot US-UK study of Cope (Osgood-Hynes et al, 1998) improved as much as similar patients did in a multisite RCT in the USA of imipramine, CBT and interpersonal therapy.

Computer guidance as a new health-care sector. Professor Steven Hirsch and myself have opened a National Health Service computer-guided self-help clinic in west London. Its broad-spectrum service may be the first of its kind. People with depression or anxiety disorder use one or more of the clinic's four self-help systems: two are PC systems - FearFighter for phobia/panic and a system for depression called Balance; the other two are IVR systems-Cope for depression and BTSteps for OCD. Clinicians screen the patients briefly by phone or face-to-face before allowing them to use a computer self-help system, and remain available for brief back-up advice as needed.

Researchers across the world are creating further computer self-help systems for mental health problems. This mode of delivering care may enable society to extend effective help at affordable cost to numerous people in the community with untreated anxiety/depression who cannot get help owing to the scarcity of suitably trained therapists, fear of stigma or difficulty in travelling to a treatment site. A host of questions arise. Which problems do and do not respond to computer-guided self-help? Who or what should screen patients for self-help, recommend and introduce them to a system, do the backup and outcome evaluation required and run computer self-help services? How can health-care systems organise such services? Can tracking mechanisms lead to an international clearing-house of clinical outcomes? Who is liable when a user of a self-help system commits suicide (which will happen eventually within any very large population even if it is just being treated for ingrown toenails)? What are the training implications for health-care professionals?

Computer-guided self-help promises to extend the availability of certain treatments and also deepen the research. Having a record of the patient's every keystroke and the time it was made could facilitate microscopic analysis of the process and mechanism of improvement.

\section{Criterion 2: only specified components of the treatment should produce the improvement}

It is one thing to show that a particular treatment package improves a given problem but it is quite another to discover which elements in that package produce the improvement and which are redundant.

Formidable problems loom in trying to define a treatment's elements. Psychotherapy lacks pharmacotherapy's advantage of centuries of research that validated ideas such as electrons, atoms, atomic weight and number, valency and molecular shape. Clomipramine differs from imipramine in having a chlorine atom in a given position. But what, if any, are the critical differences of exposure therapy from cognitive restructuring, of problem-solving from a given perspective or of task concentration from mindfulness meditation? Mendeleyev outlined the Periodic Table of chemical elements in 1869. In 2001 we still have no table of psychotherapy elements whose members are related in a single ordered system confirmed by repeated experiment.

Psychotherapy elements might not be discrete. They might overlap or be on a continuum. This may not matter. It is useful to speak of blue, green and yellow light even though they shade imperceptibly into one another along a continuum. Psychotherapy awaits a validated unifying concept like the electromagnetic spectrum, whose various wavelengths are seen as particular colours or detected in other ways.

Even the most tightly researched psychotherapies - behavioural, cognitive and interpersonal - have a tangled thicket of components. Take 'CBT' for panic. Its components differ hugely from one therapist to another, with varying mixtures of: relevant exposure (diverse forms of which have over 65 labels); interoceptive exposure (which might be a kind of stress immunisation); cognitive restructuring (including identification and monitoring of negative thoughts and changing those to positive ones); slow, deep breathing; relaxation; 
diary-keeping; particular homework exercises; family work of various forms; reward for progress; getting a treatment rationale; and expecting to improve. One therapist may use a bit of this, a lot of that and none at all of a third component from the list. Another might give none or all of those three components in equal proportion. Yet all therapists call their method CBT.

Researchers often standardise treatment by writing detailed treatment manuals. Two cheers for that. No third cheer because different manuals prescribe widely different mixtures of components. Neither do we know enough to always say which components must be in because they work well, which omitted because they are redundant and which left out because they impair efficacy.

\section{The dismantling enterprise is difficult but not impossible}

Unravelling what works in treatment is so hard a task that its value has been questioned. National Institute of Mental Health research priorities in the USA now favour treatment efficacy over mechanism, which is a mistake. Scientists have to understand both efficacy and mechanism. The fact that comparing component A with component B given for the same duration may not reveal potential higher-order effects of $A+B$ means not that dismantling should be abandoned but that $\mathrm{A}$ and $\mathrm{B}$ have to be studied eventually in combination as well as alone. Neither is it fatal to have some imprecision in defining A and B. Having many variants of serotonin receptors or of phenylketonuria genes or of AIDS viruses that mutate rapidly means that scientists must work out limiting criteria for each concept, not give up in despair.

The dismantling of psychotherapy components is not a hopeless endeavour. Hope springs from negative findings. In RCTs with anxiety disorders, certain treatment components repeatedly have helped sufferers less than others have. A therapeutic relationship is not always vital - computerguided self-help has helped with minimum or no human contact after screening. Free association usually is ineffective. Relaxation is largely redundant when done on its own and fails to enhance exposure. Anti-exposure is not helpful. Being on a waiting list does not ease chronic problems.

Until recently almost all treatments that improved anxiety disorders in RCTs involved exposure to the feared situation until fear subsided. A few RCTs found, however, that some anxiety disorders also benefited from certain approaches that used no systematic exposure or too small amounts to explain the large improvement obtained (Marks \& Dar, 2000). An example is 'pure' cognitive restructuring that excluded exposure techniques such as behavioural challenges. Whether cognitive restructuring works primarily by changing cognitions is another matter; in post-traumatic stress disorder, behaviour and symptoms improved before there was change in the beliefs that the cognitive therapy tried to alter.

Another non-exposure therapy that was effective in an RCT teaches muscle tensing exercises (without exposure or cognitive restructuring) to reduce blood phobia lastingly, perhaps by stopping bradycardic fainting at the sight of blood. A further helpful non-exposure package is problem-solving for generalised anxiety. Yet another is mindfulness meditation, which reduced stress among volunteer (Astin, 1997); such meditation contained traces of exposure but not of cognitive restructuring.

Do all anxiety-reducing methods share a common theme leading eventually onto a single road to produce improvement, or do some approaches get there by an independent route? We have to test whether exposure on its own and cognitive restructuring shorn of its behavioural elements may each be vectors for other change mechanisms that remain hidden.

Several effective treatment packages share elements such as giving sufferers perspective, teaching them to break down difficult tasks into manageable bits and giving them a sense of control. Each element needs operational definition and testing to see whether it works on its own or as a cofactor with other components, or whether it is just a product of improvement.

\section{Criterion 3: the mechanisms by which therapy components yield improvement can be delineated; Criterion 4: why therapy components help only some sufferers and not others with the same problem can be pinpointed}

According to Criteria 3 and 4, therapy is still a mewling infant with little certain to say. Given knowledge about the mechanisms of action, a mature therapeutic science would predict effects across disorders and link the effects of psychoand pharmacotherapy. Its therapists would transcend ideological differences and evolve a common language of discourse rooted in convergent experimental findings - a desire for this is emerging among researchers.

Before ending, let us put things in perspective. It took many centuries of exploration after Vikings landed in the Western hemisphere before map-makers realised that the Americas form an island distinct from Eurasia. It will take much experimentation by generations of clinical scientists to produce maps that closely mirror the world of therapy. During the 36 years since Sir Aubrey Lewis retired, therapy has become able to help certain problems and is slowly coming of age. As a science explaining that ability, however, therapy is a toddler taking its first few steps on the long road to maturity.

\section{REFERENCES}

Alkubaisy, T., Marks, I. M., Logsdail, S., et al (1992) Role of exposure homework in phobic reduction: a controlled study. Behaviour Therapy, 23, 599-622.

Astin, J. A. (1997) Stress reduction through mindfulness meditation. Psychotherapy and Psychosomatics, 66, 97-106.

Babyak, M. A., Blumenthal, J. A., Herman, S., et al (2000) Exercise treatment for major depression: maintenance of therapeutic benefit at 10 months. Psychosomatic Medicine, 62, 633-638.

Barrett, P. M., Dadds, M. R. \& Rapee, R. M. (1996)

Family treatment of childhood anxiety: a controlled trial. Journal of Consulting and Clinical Psychology, 64, 333-342.

Burgess, M., Gill, M. \& Marks, I. (1998) Postal self-exposure treatment of recurrent nightmares. Randomised controlled trial. British Journal of Psychiatry, I72, 257-262.

Dadds, M. R., Spence, S. H., Holland, D. E., et a (1997) Prevention and early intervention for anxiety disorders: a controlled trial. Journal of Consulting and Clinical Psychology, 65, 627-635.

Fava, G. A., Rafanelli, C., Grandi, S., et al (1998) Six-year outcome for CBT of residual symptoms in major depression. American Journal of Psychiatry, I55, 1443-1445.

_, _ , _ , et al (200la) Long-term outcome of panic disorder with agoraphobia treated by exposure. Psychological Medicine, 31, 891-898.

_ , Grandi, S., Rafanelli, C., et al (200lb) Long-term outcome of social phobia treated by exposure. Psychological Medicine, 31, 899-905.

Frank, E. \& Spanier, C. (1995) Interpersonal psychotherapy for depression: overview, clinical efficacy, and future directions. Clinical Psychology: Science and Practice, 2, 349-369.

Ghosh, A., Marks, I. M. \& Carr, A. C. (1988)

Therapist contact and outcome of self-exposure treatment for phobias. A controlled study. British journal of Psychiatry, 152, 234-238. 
Ginsberg, G., Marks, I. M. \& Waters, H. (1984) Cost benefit analysis of a controlled trial of nurse therapy in primary care. Psychological Medicine, 14 683-690.

Gortner, E.T., Gollan, J. K., Dobson, K. S., et al (1998) Cognitive-behavioral treatment for depression: relapse prevention. Journal of Consulting and Clinical Psychology, 66, 377-384.

Greist, J. H., Marks, I. M., Baer, L., et al (2002) Behavior therapy for obsessive-compulsive disorder guided by a computer or by a clinician compared with relaxation as a control. Journal of Clinical Psychiatry, 63, 138-145.

Jacobson, N. S., Dobson, K. S., Truax, P. A., et al (1996) A component analysis of cognitive-behavioural treatment for depression. Journal of Consulting and Clinical Psychology, 64, 295-304.

\section{Leff, J., Vearnals, S., Brewin, C. R., et al (2000)} The London Depression Intervention Trial. Randomised controlled trial of antidepressants $v$. couple therapy in the treatment and maintenance of people with depression living with a partner: clinical outcome and costs. British Journal of Psychiatry, 177, 95-100.

Marks, I. M. (1987) Fears, Phobias, and Rituals. New York: Oxford University Press.

— \& Dar, R. (2000) Fear reduction by psychotherapies. Recent findings, future directions. British Journal of Psychiatry, 176, 507-5II.

Osgood-Hynes, D. H., Greist, J. H., Marks, I. M., et al (1998) Self-administered psychotherapy for

depression using a telephone-accessed computer system plus booklets: an open US-UK study. Journal of Clinical Psychiatry, 58, 358-365.

O'Sullivan, G. \& Marks, J. M. (1990) Long-term outcome of phobic and obsessive-compulsive disorders after treatment. In Handbook of Anxiety (eds R. Noyes, M. Roth \& G. D. Burrows), pp. 87-108. Amsterdam: Elsevier.

\section{CLINICAL IMPLICATIONS}

Brief therapy is mature in its ability to improve anxiety and depressive disorders reliably and enduringly, even when guidance is by computer self-help.

- More work is needed on brief therapy's cost-effectiveness and widening its acceptability still further.

- Therapy is in its infancy regarding which of its components produce improvement, how they do so and why some sufferers do not respond.

\section{LIMITATIONS}

Disorders other than anxiety and depression are not considered.

- Interactions with medication are not discussed.

- The prediction of future trends is not attempted.

ISAAC M. MARKS, FRCPsych, Institute of Psychiatry, London SE5 8AF, UK. E-mail: I.Marks@iop.kcl.ac.uk

(First received 22 December 2000, final revision 26 April 200I, accepted 27 April 200I)

Selmi, P. M., Klein, M. H., Greist, J. H., et al (1990) Computer-administered CBT for depression. American Journal of Psychiatry, 147, 5I-56.

Shepherd, M. (1991) Sir Aubrey Lewis - an Australian psychiatrist. In Australian Psychiatry and the Tradition of Aubrey Lewis (ed. D. Copolov), p. II. Melbourne:
NHMRC Schizophrenia Research Unit, Monash and Melbourne Universities.

Teasdale, J. D., Segal, Z.V., Williams, J. M., et al (2000) Prevention of relapse/ recurrence in major depression by mindfulness-based cognitive therapy. Journal of Consulting and Clinical Psychology, 68, 615-623. 